\title{
MILLION DOLLAR LOGISTIC DECISIONS USING SIMULATION
}

\author{
Michael Carr \\ Center for Entrepreneurial Studies and \\ Development, Inc. \\ Affiliated with West Virginia University \\ Morgantown, West Virginia 26506-6107, U.S.A.
}

\author{
Howard Way \\ Tropicana Products, Inc. \\ $100113^{\text {th }}$ Avenue East \\ Bradenton, Florida 34208, U.S.A.
}

\begin{abstract}
This paper presents the development of a flexible simulation model of the rail operations at the Tropicana facility in Bradenton, Florida. The complexity of the process and system interaction required a dynamic simulation model to be developed. The model describes the loading, staging, travel, and unloading of rail cars at the Tropicana facility and two distribution centers. The model output and sensitivity analysis enabled management to optimize rail car availability and crew sizing.
\end{abstract}

\section{INTRODUCTION}

Currently Tropicana ships unit trains from Bradenton Florida to a regional distribution center in the Northeast. Growth has dictated additional rail traffic to a distribution center in the Midwest. This equates to over 250 rail car deliveries every week. Maintaining and monitoring of the system is a logistics nightmare. Maintaining superior levels of customer service have become increasingly difficult. Product demand fluctuations resulted in frequent scheduling and transportation changes.

Several spreadsheet models have been developed to assist schedulers in the formidable task of locating, queuing, loading, and moving the appropriate rail cars. These models were not able to capture the system interaction and interdependency that is inherent in a system of this magnitude. The rail simulation was developed to represent the entire rail operation originating with rail car loading through transport to and from the distribution centers, and completing the cycle at either the maintenance facility or the return to the originating rail yard in Bradenton.

\section{PROBLEM DEFINITION}

\subsection{Objectives}

Tropicana developed the simulation model as a tool to allow management to analyze and understand future capital expenditures to support product growth and delivery. Additionally, capacity requirements in the areas of rail car availability, dock space at Bradenton, and crew loading requirements had been raised. Ultimately the model will be expanded to simulate the warehousing and distribution section of the process.

\subsection{Scope}

The model simulates the rail operation at Tropicana, Bradenton and two distribution centers. The model was built to evaluate the entire rail operations through delivery and return to the distribution centers (DC).

The model start point is with rail cars being loaded based on a fixed delivery schedule. Trains leave for our Northeast distribution center on Sunday, Tuesday, and Friday. Trains leave for our Midwest distribution center on Monday and Wednesday. Cars are requested at the dock 48 hours prior to loading ( if available). Rail crews load cars based on schedules/pick lists provided. As cars are loaded they are sealed and returned to the Bradenton yard to await a full train - 60 cars for the Northeast DC, 30 cars for the Midwest DC - and shipment to their respective destinations. Trains travel to the distribution center based on the above mentioned days. Travel time to each DC is reasonably fixed (events such as derailments or weather-related delays impact delivery times). As cars arrive at the distribution center they are checked in and unloaded; first priority is given to "needed" product, last priority is given to other products as space opens up in the warehouse. Unloaded cars are staged at the distribution center and wait until a specific "pull" time by CSX, i.e., all empty cars that are ready for return by 4:00 p.m. are 
pulled by CSX. The return trip to Bradenton is again based on a fixed schedule provided by CSX. Upon their return, a percentage of the cars go to rail car maintenance ( this probabilistic branch was based on historical data). These cars remain at maintenance for a distributed time and return to the yard for loading. The remaining cars enter the Bradenton yard directly. All cars have routine maintenance and fueling performed and then become available to be loaded.

\section{MODEL DEVELOPMENT}

The rail simulation was done using Process Model from ProModel Corporation. Process Model is a flow chart based simulation system which uses ABC FlowCharter from Micrografx as the user interface, and ProModel's simulation engine for the actual running of the simulation. This combination had some benefits, but was not without some tradeoffs.

\subsection{Model Organization}

In order to facilitate model development, the model was divided into two files:

- The main file included the overall system from Bradenton, Florida to the two regional distribution centers.

- The second file contained the detailed operations of the loading dock in Bradenton, Florida.

Because the number of rail cars available to Tropicana was fixed, the model was designed as a closed system, i.e., once the total number of cars were generated by the model, they remained in the system for the duration of the simulation.

\subsection{Evaluation of Benefits}

\subsubsection{Short Learning Curve}

Simulation is by no means a trivial task. It requires a thorough knowledge of queuing theory, statistics, and process modeling. This aside, if the reader is familiar with a general purpose simulation language or other discrete event simulation software, then making the transition to ProcessModel is very easy. Each flow chart symbol is treated as an object that has various parameters to be set by the developer. Custom code can be attached to each object to allow for user specified behaviors.

\subsubsection{Ease of Transfer from Developer to User}

A dual benefit of an easy-to-learn simulation package is the ease at which the model can be transferred from developer to user. Users familiar with simulation, can quickly get up to speed on the use of ProcessModel and can focus the majority of their time on learning the specifics of the particular business process being modeled.

An easy-to-learn simulation package has the added benefit of a reduced dependency on the developer for many of the future scenario changes that were not predicted when the model was developed.

\subsubsection{Hierarchical Modeling}

Though ProcessModel is a flow chart based simulation package, it has some very powerful features. One of those features is the ability to do enterprise modeling. This is accomplished through a hierarchical modeling feature. Each activity on a flow chart can have a more detailed sub-model describing its behavior. Hierarchical modeling allows you to link separate business model flowcharts into a larger enterprise model. This feature was used to link the detailed operation of the Bradenton docks to the overall flow model that included the distribution centers.

\subsubsection{Compatibility with ProModel}

Models developed in ProcessModel are upwardly compatible with ProModel. This allows for an easy upgrade path for those unsure about the benefits of simulation or unwilling to make the capital investment needed to purchase a large-scale simulation software package.

\subsection{Evaluation of Trade-offs}

Though ProcessModel is easy to learn and use, it is not without some trade-offs.

\subsubsection{Interface Implementation}

The most immediate trade-off was the design time interface speed. $\mathrm{ABC}$ FlowCharter and ProModel communicate through an OLE (Object Linking and Embedding) interface. This process consumes a large amount of computing resources, particularly random access memory. Initial development took place on an Pentium $75 \mathrm{MHz}$ computer with the minimum recommended memory of $16 \mathrm{Mb}$. Under these conditions, ProcessModel performed slowly, often leaving the authors wondering if any processing was 
taking place. Upgrading the memory to $40 \mathrm{Mb}$ helped improve the interface speed substantially. Model run time speed was adequate, and seemed unaffected by the additional memory.

\subsubsection{Graphical Representation of Model}

A big trade-off on the visual front was the lack of graphics appropriate to simulate a rail distribution system. Custom graphics can be created and substituted for the graphics supplied with ABC FlowCharter for every object, except entities. Entities must use the predefined graphics supplied with the software. In the case of the rail simulation, we settled on a freight truck to represent a train in the model.

\subsubsection{Model Flow Control}

Because the model was designed as a closed system, flow control became a critical feature of the model. To accurately replicate the schedules and rules used for the movement of trains, interesting control flow charts had to be built to control these situations.

\subsubsection{Resource Scheduling}

The amount of resources available for an activity in a model can be easily adjusted through a resource attribute called quantity. If you want to have two crews load trains, for example, simply change the crew resource quantity from 1 to 2 and rerun the model. This method of resource quantity adjustment works flawlessly until a resource availability schedule or shift schedule is attached to the resource. When a schedule is attached to a resource with a quantity greater than one, only the first resource abides by the schedule. The remaining resources are available 24 hours per day, 7 days per week. To get around this problem, separate single resources were added to the model for each scheduling scenario. This made schedule change scenarios inconvenient at best.

\section{MODEL DESCRIPTION}

\subsection{Model Features}

The simulation is designed to model the flow of rail cars from the rail yard in Bradenton to the two distribution centers and back to Bradenton. See Figure 1.

\subsection{Model Outputs}

Various statistics and outputs collected from the rail simulation are listed below:
- Distribution of the number of chilled and ambient rail cars available in the yard.

- Crew utilization at the Bradenton docks, Northeast distribution center, and the new Midwest distribution center.

- Dock utilization.

- CSX train switching needs.

- Cycle time of cars through various points in the system.

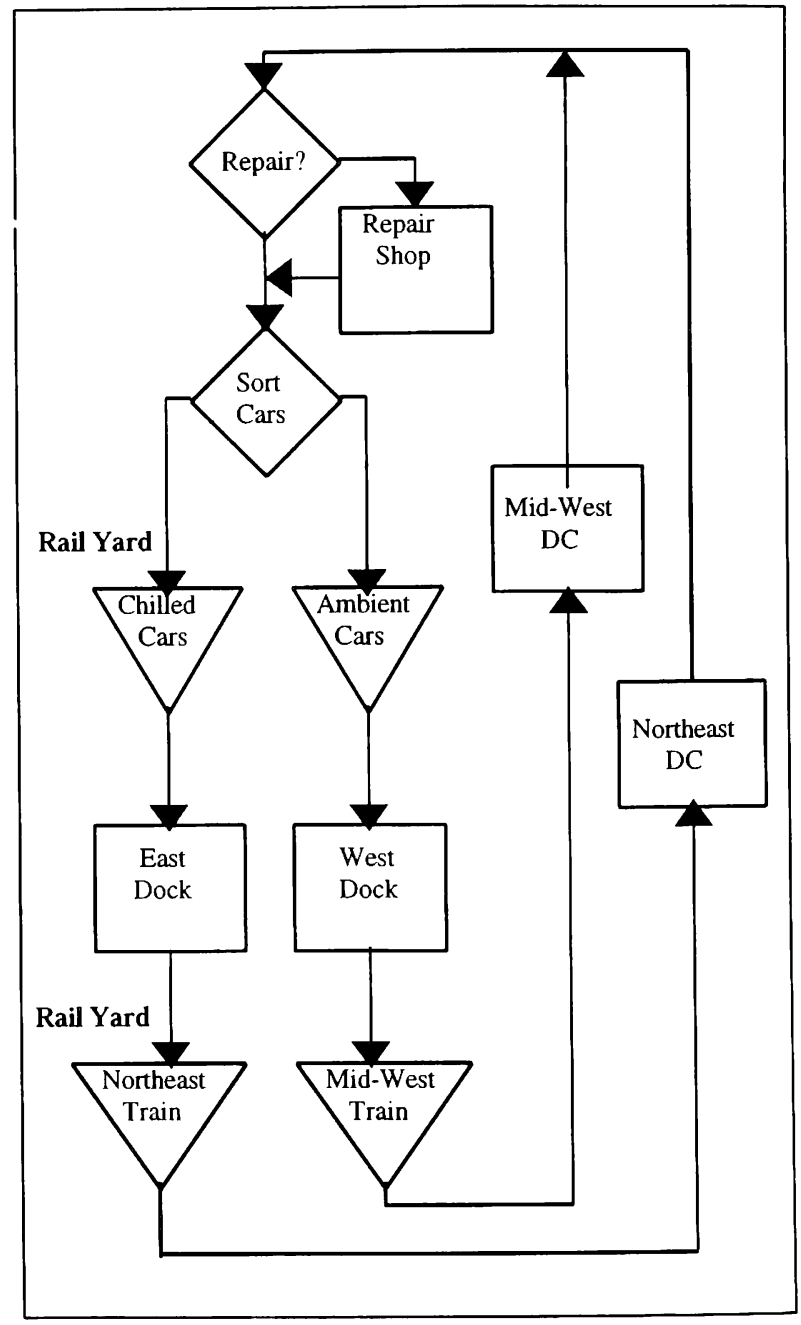

Figure 1: Rail Operations Flow Chart

\section{MODELING VALIDATION \& RESULTS}

\subsection{Validation}

The model was initially validated with the Northeast distribution center only. The reason was this DC had been in operation for several years and historical data was readily available. (The Midwest DC had been in operation for less than 1 month). The model was run with existing train schedules, crew and car availability, 
and warehouse unloading times. These results were presented to traffic/scheduling personnel within the rail department. Our car availability results, i.e., how many cars at any given day were available at the Bradenton yard, was within 3 to 5 cars daily. (The total available fleet for Tropicana is 415 cars). The Midwest DC was then added and results were again validated and checked with the traffic department. The results again confirmed our high level of duplication of the current system. To validate crew availability, utilization statistics were compared to labor reports.

\subsection{Results}

\subsubsection{Rail Car Utilization}

Several scenarios were run to determine the impact of rail car cycle times between Bradenton and the distribution centers on rail car utilization. Since the majority of the cars are sent to the Northeast DC, emphasis was placed on reducing rail car travel time for that portion of the model. Discussions with CSX personnel identified potential no-cost areas for travel time reduction. These times were individually entered into the model and the resulting impact was determined. Combinations of anticipated travel-time scenarios were entered. The model identified those areas that had the greatest impact on overall rail car availability.

\subsubsection{Crew Loading Requirements at Bradenton}

Current crewing to satisfy the Northeast distribution center was used in the base model at current demand levels. Twelve different scenarios were run to evaluate crew loading, i.e., different shifts, different work schedules. The model identified additional crews would be needed to satisfy the Midwest demand.

To determine the optimum crew assignments, the number of discharge points from the warehouse was held at the current level and the number of rail spots was also kept constant. The highest crew utilization and system throughput occurred for crews utilized over 2 shifts.

\subsubsection{Crew Unloading Requirements at the Distribution Centers}

Unloading times had been entered into the model based on historical data from each distribution center. These times, i.e., schedules and shifts were varied to determine overall system impact. The model helped identify potential shift variations to increase rail car availability and throughput.

\section{SUMMARY}

The model allowed Tropicana to evaluate many loading and delivery schedules; understanding their impact before actual implementation. It was determined that we did not need to add more rail cars to the $400+$ fleet in order to meet the increased demand of rail cars by the Midwest distribution center. Asset utilization has increased with a bottom line impact of $\$ 4 \mathrm{MM}+$ of avoided new investment. Customer service measures have improved and overall system costs have been avoided. The model will be expanded as future business demands dictate.

\section{ACKNOWLEDGMENTS}

Each author contributed equally to this paper.

\section{AUTHOR BIOGRAPHIES}

MICHAEL C. CARR is a partner with Computer Companion, Inc., and a Contract Associate with the Center for Entrepreneurial Studies and Development, Inc. He received B.S. and M.S. degrees in industrial engineering from West Virginia University in 1987 and 1995 respectively. $\mathrm{He}$ is a member of Alpha Pi Mu, INFORMS, IIE, and the American College of Health Care Executives.

HOWARD WAY is a Senior Project Manager with Tropicana Products, Inc., specializing in distribution and warehousing. Before joining Tropicana, he was an industrial engineer with Anheuser-Busch, Inc. He received B.S. and M.S. degrees in industrial engineering from Rochester Institute of Technology in 1982 and 1985 respectively. He is a member of IIE. 